\title{
CORRECTION
}

\section{Correction: One-step fabrication of robust fabrics with both-faced superhydrophobicity for the separation and capture of oil from water}

Cite this: Phys. Chem. Chem. Phys., 2015, 17, 11112

\author{
Jian Li, ${ }^{*}$ Long Yan, Yuzhu Zhao, Fei Zha, Qingtao Wang and Ziqiang Lei
}

DOI: $10.1039 / c 5$ cp90057c

Correction for 'One-step fabrication of robust fabrics with both-faced superhydrophobicity for www.rsc.org/pccp the separation and capture of oil from water' by Jian Li et al., Phys. Chem. Chem. Phys., 2015, 17, 6451-6457.

Fig. 6 in the published article is incorrect. Fig. 6 should be as follows:
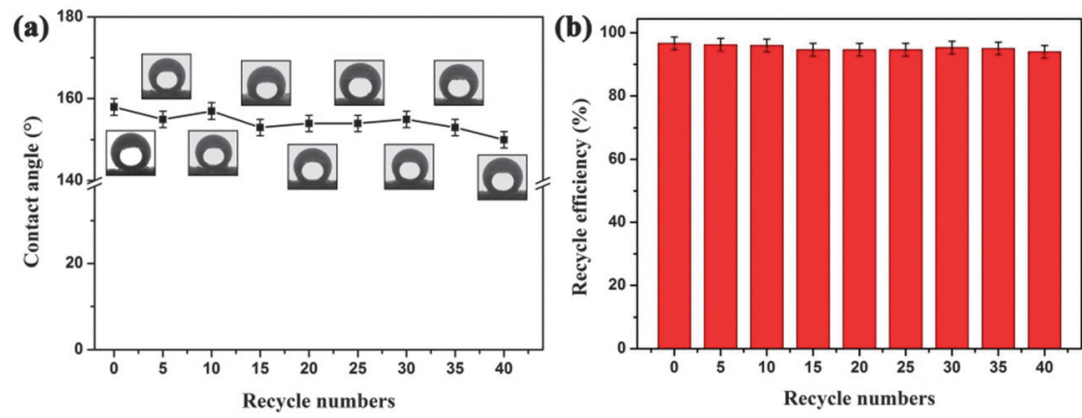

The Royal Society of Chemistry apologises for these errors and any consequent inconvenience to authors and readers. 